\title{
Développement d'une cellule robotisée de détourage des composites
}

\author{
Claire Dumas, Aude Boudelier ${ }^{a}$, Stéphane Caro, Sébastien Garnier, \\ Mathieu Ritou et Benoît Furet \\ Institut de Recherche en Communications et Cybernétique de Nantes, UMR CNRS n 6597,1 rue de la Noë, \\ 44321 Nantes, France
}

Reçu le 6 décembre 2010, accepté le 27 avril 2011

\begin{abstract}
Résumé - L'usinage robotisé, nouveau challenge de la robotique industrielle, nécessite à la fois rigidité et précision du moyen de production. C'est pourquoi cette pratique particulière du détourage des composites était réservée jusqu'alors aux machines-outils à commande numérique. Cet article propose de démontrer, qu'à partir d'une connaissance des phénomènes de coupe liés aux procédés et de la modélisation des caractéristiques comportementales du robot, il est possible d'optimiser l'exploitation des cellules robotisées. Cette première étude porte sur le cas spécifique du détourage des pièces composites. D'un côté, le robot a été modélisé et ses paramètres de raideur ont été identifiés. De l'autre côté, les conditions de coupe optimales permettant de respecter l'intégrité matière et d'assurer la productivité souhaitée ont été déterminées. Les efforts d'usinage ont alors été relevés. À partir de ces deux études parallèles, les déformations du robot, pour un placement donné de la pièce dans son espace de travail, ont pu être déterminées. Par la suite, ces déformations peuvent être limitées en plaçant judicieusement la tâche dans le volume de la cellule robotisée. Cette solution simple permet de réduire significativement les déformations observées, notamment dans le cas de procédés sollicitant fortement le robot, et ce sans modifier sa commande.
\end{abstract}

Mots clés : Robotique d'usinage / placement de tâches / détourage / effort d'usinage / matériau composite

\begin{abstract}
Development of a robotic cell for trimming of composite parts. Robot machining is a new challenge in robotics as it requires both high stiffness and accuracy of the robot at hand. Until now, machining operations have been mainly realized with numerical-control machine-tools. This paper pertains to the optimization of the use of industrial robots for finishing tasks knowing the process cutting phenomena and the robot stiffness. Composite parts trimming is used as an illustrative machining operation in the framework of this paper. On the one hand, the robot was modeled and its joint stiffness values were identified. On the other hand, tests for composites parts trimming were performed in order to determine optimal cutting conditions guaranteeing the integrity (a good quality of) the part and satisfying productivity. Moreover, cutting forces were measured during the tests thanks to a wrench sensor mounted on the robot end-effector. From those two parallel studies and from a given placement of the part into the robot workspace, the robot end-effector displacements can be determined. Then those displacements could be minimized by determining the best placement of the part into the robot workspace. For operations that hardly stress the robot this solution can significantly reduce the robot end-effector displacements, without modifying the robot control.
\end{abstract}

Key words: Robotic machining / tasks placement / trimming / cutting force / composite material

\section{Introduction}

Conçus à l'origine pour des opérations de « pick and place », les robots industriels ont aujourd'hui évolué

\footnotetext{
a Auteur pour correspondance :

aude. boudelier@irccyn.ec-nantes.fr
}

et sont utilisés pour des applications plus diversifiées (soudage, peinture, assemblage...). Ces manipulateurs ont été développés soit pour aider l'opérateur dans certaines tâches, soit pour prendre en charge des opérations réalisées sur machines-outils. Dans le premier cas, ils permettent de diminuer le taux de rebut et le coût des pièces produites, d'augmenter le volume de production ainsi que 


\section{Nomenclature}

\begin{tabular}{|ll|}
\hline$\delta \mathbf{d}$ & Déplacements du « tool center point $»(\mathrm{TCP})$ du robot, exprimés dans $\mathcal{F}_{0}$ \\
$\mathcal{F}_{0}$ & Référentiel de base du robot, selon la convention de Denavit Hartenberg modifiée \\
$F_{\mathrm{f}}$ & Effort au niveau de l'effecteur \\
$F_{n}$ & Force normale \\
$F_{z}$ & Force axiale \\
$\mathbf{J}$ & Matrice jacobienne cinématique du robot \\
$k_{\theta_{i}=1 . .6}$ & Raideurs articulaires \\
$\mathbf{K}_{C}$ & Matrice de raideur complémentaire du robot \\
$\mathbf{K}_{\theta}$ & Matrice de raideur articulaire du robot \\
$\mathbf{K}_{X}$ & Matrice de raideur cartésienne du robot \\
$\boldsymbol{\omega}$ & Forces et moments appliqués sur le TCP du robot, exprimés dans $\mathcal{F}_{0}$ \\
$\theta_{i=1 . .6}$ & Coordonnées articulaires \\
\hline
\end{tabular}

la flexibilité de la chaîne. Le deuxième cas concerne plus particulièrement les applications pour lesquelles, au regard de la qualité, l'investissement de machines-outils n'est pas toujours justifié. Les robots industriels sont certes moins précis, mais à iso-volume de travail, ils sont trois à quatre fois moins chers. C'est pourquoi plus la pièce est de grandes dimensions, plus l'utilisation de ces robots se justifie.

En parallèle, des domaines tels que l'aéronautique et le nautisme, à travers l'utilisation des matériaux composites, réalisent aujourd'hui de plus en plus de pièces de grandes dimensions. Le procédé de fabrication de ces pièces contient une étape de finition, dite de parachèvement [1]. Elle est nécessaire afin de respecter les tolérances dimensionnelles spécifiées et les qualités de surface exigées $[2,3]$. Souvent réalisée manuellement, cette opération se traduit par un manque de précision et un taux de rebut important.

La robotisation permettrait donc de rendre la production de ces pièces plus fiable et moins onéreuse. Cependant, il n'existe pas de méthode d'implantation réellement adaptée pour ces robots usineurs. Nous proposons une approche scientifique construite autour du couplage de la modélisation des caractéristiques comportementales du robot et du procédé. Cet article développe cette démarche pour une opération de détourage robotisée d'une pièce composite.

\section{Capabilité des robots sériels lors d'opérations d'usinage}

Aujourd'hui, les constructeurs s'intéressent de près aux opérations d'usinage et développent de nouvelles solutions pour ces applications. Néanmoins, il n'existe pas à l'heure actuelle de solution robotisée fiable, utilisable pour tous les procédés d'usinage.

\section{1 Évaluation expérimentale des capacités des robots à effectuer des opérations d'usinage}

Afin d'évaluer la capacité d'un robot à effectuer des opérations d'usinage, des essais ont été réalisés sur un robot ABB 6 axes (Fig. 1a). Sa capacité de charge est de $205 \mathrm{~kg}$ et son rayon d'action est de 1,93 m. Son espace de travail est représenté par la figure 1b. Les opérations réalisées sont présentées dans le tableau 1.

À l'issue de ces essais, les pièces obtenues ont été mesurées. Les résultats montrent des écarts géométriques importants (jusqu'à 1,3 mm). De plus, le niveau RMS augmente très rapidement en fonction de l'engagement axial. Ces premiers essais ont montré qu'un robot, utilisé comme une machine-outil, avait de grandes difficultés à respecter les spécifications géométriques souhaitées. Il est donc nécessaire d'étudier son comportement et d'adapter l'opération d'usinage à ses caractéristiques.

\subsection{L'usinage robotisé}

L'idée de pouvoir usiner avec un robot n'est pas nouvelle. Malgré une dizaine d'années d'études à ce sujet, il n'y a pas encore de méthode fiable permettant de réaliser de telles opérations tout en conservant la flexibilité importante qui constitue l'atout majeur d'un robot sériel par rapport à une machine à commande numérique. Dorénavant, il est possible d'usiner avec des robots à structure parallèle [4]. Mais ces manipulateurs présentent l'inconvénient d'avoir un faible ratio espace de travail/empreinte au sol ainsi qu'une accessibilité limitée par rapport aux robots industriels sériels $6 \mathrm{R}$. C'est pourquoi les recherches effectuées ici se concentrent spécifiquement sur ce type de manipulateur.

Différentes études ont permis d'identifier les phénomènes limitant l'usinage par un robot industriel. Le plus pénalisant est leur manque de raideur, souvent cause du non-respect des tolérances géométriques [5-8]. Comparativement à une machine-outil standard, la raideur du robot est environ cinquante fois plus faible [6]. Matsuoka [5] a étudié les caractéristiques de l'usinage robotisé. Il propose d'améliorer la précision obtenue en diminuant les efforts de coupe, et ce par l'utilisation d'un outil de faible diamètre tournant à une vitesse élevée. Zhang [6] propose une méthode de compensation en temps-réel de la déflection du manipulateur par un calcul des déformations instantanées de chaque articulation. Pour cela, un modèle de raideur est identifié puis alimenté par la mesure en continu de l'effort exercé sur le robot. 


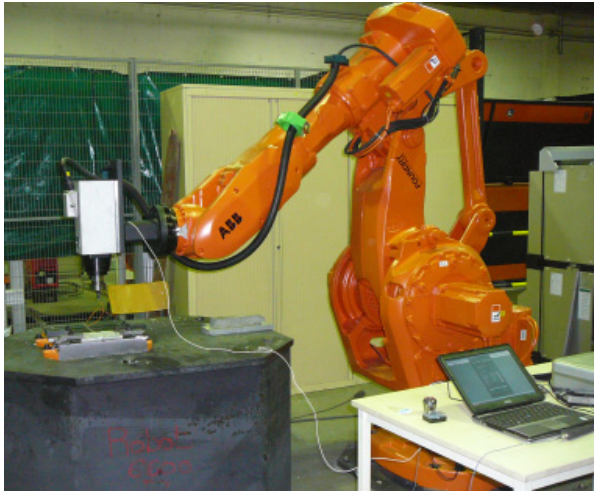

(a)

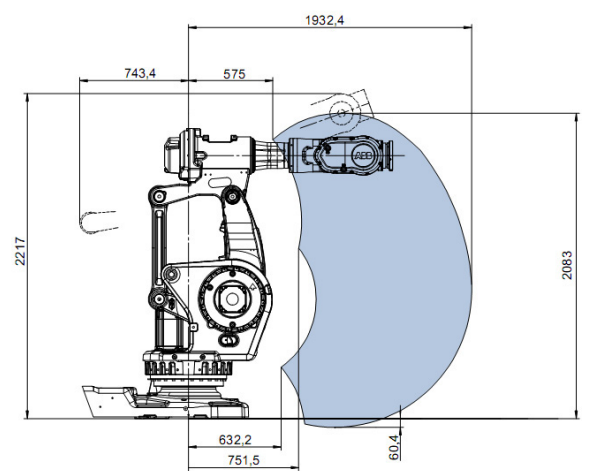

(b)

Fig. 1. Robot ABB IRB 6660 (a) et son espace de travail (b).

Tableau 1. Conditions des essais.

\begin{tabular}{cccc}
\hline Matière usinée & \multicolumn{2}{c}{ Alliage d'aluminium aéronautique (série 7000) } \\
Outil utilisé & Fraise carbure Fraise diam.16 L92 Z2 (Réf 5272610) \\
Vitesse de rotation [tr.min ${ }^{-1}$ ] & \multicolumn{4}{c}{12000} \\
Avance par tour [mm.tr ${ }^{-1}$ ] & \multicolumn{3}{c}{0,2} \\
Engagement radial $[\mathrm{mm}]$ & 0,5 à 3 & 3 & 2,5 \\
Engagement axial $[\mathrm{mm}]$ & Rainurage & Rainurage & Zig-Zag \\
Sens d'usinage dans le & selon X+ & selon Y+ et Y- & en X+, Y+, puis X- \\
référentiel robot &
\end{tabular}

Ainsi, les déplacements de l'outil peuvent être déduits. Enfin, Olabi [8] a montré qu'en générant des trajectoires douces à jerk limité, il est possible de diminuer fortement les erreurs de précision.

Le deuxième phénomène limitant identifié est la vibration de la structure même du robot $[7,9]$. Comparativement à une machine-outil standard, la fréquence propre d'un robot est 10 à 100 fois plus faible [9]. Pan a montré que le phénomène vibratoire constaté lors de l'usinage avec un robot n'était pas de nature « régénérative » mais structurelle [9]. Par une modélisation conjointe du robot et des efforts de coupe, un critère de stabilité a été développé puis testé. Enfin, à partir de plusieurs essais expérimentaux, des règles pour l'usinage robotisé ont été proposées.

Ces différentes études proposent des pistes d'amélioration pour l'usinage à l'aide de robot industriel 6R. Dans cet article, une nouvelle méthode est proposée, permettant de prédire à partir des efforts exercés sur le robot, les déformations de l'effecteur en rotation et en translation. Des pistes sont ensuite données afin de les limiter sans devoir modifier la commande du robot. Effectivement, cette tâche nécessiterait une étape d'adaptation pour chaque robot de marque différente.

\section{Identification des paramètres de raideurs des robots $6 R$}

Connaissant l'effort auquel l'effecteur du robot est soumis, les déplacements du robot peuvent être prédits. Pour cela, un modèle de raideur basé sur l'hypothèse 《bras rigides-articulations flexibles » a été utilisé [10]. Il permet de relier les efforts exercés sur l'effecteur aux déplacements de ce dernier via la matrice de raideur cartésienne $\mathbf{K}_{X}$ :

$$
\boldsymbol{\omega}=\mathbf{K}_{X} \delta \boldsymbol{d}
$$

avec $\boldsymbol{\omega}$ le vecteur $6 \times 1$ des efforts et moments exercés sur l'effecteur du robot, $\delta \boldsymbol{d}$ le vecteur $6 \times 1$ des déplacements linéaires et angulaires de l'effecteur.

La matrice de raideur cartésienne $\mathbf{K}_{X}$ depend de la configuration du robot, des raideurs de chaque articulation et des efforts et moments exercés sur l'effecteur, selon la relation :

$$
\mathbf{K}_{X}=\mathbf{J}^{-T}\left(\mathbf{K}_{\theta}-\mathbf{K}_{C}\right) \mathbf{J}^{-1}
$$

avec

$$
\mathbf{K}_{\theta}=\left[\begin{array}{cccccc}
k_{\theta_{1}} & 0 & 0 & 0 & 0 & 0 \\
0 & k_{\theta_{2}} & 0 & 0 & 0 & 0 \\
0 & 0 & k_{\theta_{3}} & 0 & 0 & 0 \\
0 & 0 & 0 & k_{\theta_{4}} & 0 & 0 \\
0 & 0 & 0 & 0 & k_{\theta_{5}} & 0 \\
0 & 0 & 0 & 0 & 0 & k_{\theta_{6}}
\end{array}\right]
$$

et

$$
\mathbf{K}_{C}=\left[\frac{\partial \mathbf{J}^{T}}{\partial \theta_{1}} \omega \frac{\partial \mathbf{J}^{T}}{\partial \theta_{2}} \omega \frac{\partial \mathbf{J}^{T}}{\partial \theta_{3}} \omega \frac{\partial \mathbf{J}^{T}}{\partial \theta_{4}} \omega \frac{\partial \mathbf{J}^{T}}{\partial \theta_{5}} \omega \frac{\partial \mathbf{J}^{T}}{\partial \theta_{6}} \omega\right]
$$

$\mathbf{J}$ étant la matrice jacobienne du manipulateur. La matrice $\mathbf{K}_{\theta}$, diagonale, est constituée des raideurs de chaque articulation [Nm.rad $\left.{ }^{-1}\right] . \mathbf{K}_{C}$, appelée matrice de raideur complémentaire, permet de prendre en compte l'influence des efforts sur la raideur du manipulateur. 


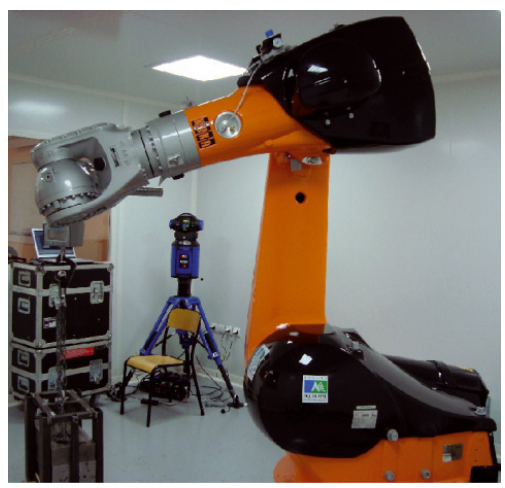

Fig. 2. Identification des raideurs apparentes du robot.

Nous avons développé un protocole d'identification permettant, à partir d'un nombre raisonnable d'essais, de déterminer la matrice $\mathbf{K}_{\theta}$ [11]. Contrairement aux méthodes existantes, les raideurs de tous les axes peuvent être identifiées, ce qui rend possible le calcul des déplacements de l'effecteur, à la fois en translation et en rotation. Enfin, ce protocole permet d'intégrer en partie la flexibilité des bras dans les raideurs articulaires par l'identification de raideurs « apparentes ». Ainsi, les raideurs du robot KR240-2 (Fig. 2) ont été identifiées (Tab. 2).

\section{Détourage d'une pièce composite : efforts générés}

\subsection{Usinage des matériaux composites}

De part leur nature hétérogène, l'usinage des matériaux composites est très différent de l'usinage des matériaux métalliques. Effectivement, leurs propriétés mécaniques et thermiques les rendent difficiles à parachever : anisotropie, faible conductivité thermique, niveau élevé d'abrasion $[12,13]$.

Les outils coupants utilisés usuellement pour usiner les matériaux métalliques atteignent aujourd'hui leur limite dans le domaine du composite. Par exemple, les outils carbures permettent d'obtenir une bonne qualité. Cependant, leur durée de vie est très courte en raison du caractère abrasif des matériaux composites [14]. Ainsi, les PCD (Poly Cristallin Diamond) sont apparus comme une alternative intéressante, alliant amélioration de la durée de vie, de la vitesse d'avance et de la qualité générée $[12,15,16]$. Néanmoins, leur coût est relativement élevé et leur usure toujours prématurée. C'est pourquoi des outils diamantés, de plus en plus utilisés industriellement, ont été développés [17]. Leur faible coût et leur résistance à l'abrasion en font des atouts majeurs qui permettent aujourd'hui d'allonger la durée de vie des outils et d'améliorer la productivité [18]. De plus, la géométrie de coupe multi-grains de ces outils particuliers, proche du domaine des meules, permet d'avoir des niveaux vibratoires très faibles en usinage.

Quelle que soit la nature des outils coupants, les efforts d'usinage créés sont relativement importants. Afin de répondre aux critères d'intégrité matière et d'état de surface requis, il est nécessaire d'utiliser des outils et des conditions de coupe adaptés à l'application donnée. Effectivement, en cas de mauvais choix, des défauts non acceptables, tels que le délaminage de type 1 et 2 [12], apparaissent sur les contours détourés en raison d'efforts de coupe trop importants qui désolidarisent les différents plis des composites laminés. Il est donc nécessaire de connaître le niveau d'efforts acceptable pour ne pas endommager la pièce à parachever.

De nombreux modèles d'efforts d'usinage ont été proposés dans le domaine de l'usinage des matériaux métalliques [19-22]. Ces derniers nécessitent cependant des modifications afin d'être adaptés aux matériaux composites. Effectivement, les efforts d'usinage dépendent notamment de l'orientation des fibres par rapport à la direction de l'avance. En fonction de la trajectoire réalisée, même si l'épaisseur et la largeur de coupe restent constantes, les efforts d'usinage varient. C'est pourquoi de nouveaux modèles de coupe, prenant en compte ces considérations, ont été proposés [23, 24].

Afin d'étudier la faisabilité d'une opération de détourage de pièce composite à l'aide d'un outil diamanté monté sur un système robotisé sériel, des essais préliminaires ont été effectués sur une MOCN pour quantifier le niveau des efforts d'usinage créés. Ces essais ont permis de déterminer des conditions de coupe et des paramètres outils répondant aux critères de qualité souhaités.

\subsection{Essais préliminaires}

\subsubsection{Protocole expérimental}

Un composite à base de matrice époxy et de fibres de carbone a été utilisé pour réaliser ces essais préliminaires de détourage. Des rainures ont été usinées dans des éprouvettes de 10,5 mm d'épaisseur obtenues préalablement à partir d'un procédé de drapage. Les 40 plis de pré-imprégnés ont été alternés suivant quatre orientations différentes : $0^{\circ},-45^{\circ},+45^{\circ}$ et $90^{\circ}$. Les propriétés mécaniques de ce matériau sont précisées dans le tableau 3.

Les essais ont été menés sur une machine d'usinage à grande vitesse composée de 3 axes (Huron, modèle KX30, de la plate-forme UGV d'Europe Technologies). La puissance disponible à la broche Kessler est de $40 \mathrm{~kW}$ et la vitesse maximum de rotation est de $28000 \mathrm{tr}$. $\mathrm{min}^{-1}$. Afin de mesurer les différents efforts d'usinage, une table dynamométrique à trois composantes (Kisler, type 9255B) a été positionnée entre le montage d'usinage et la table de la machine. Un système de lubrification interne et externe à l'outil a été mis en oeuvre. La figure 3 montre le dispositif mis en place pour la réalisation de ces essais préliminaires.

Comme précisé précédemment, afin de limiter les défauts créés par les efforts d'usinage, deux types de paramètres ont été testés afin de déterminer les plus 
Tableau 2. Raideurs apparentes du robot en $\mathrm{Nm} \mathrm{rad}^{-1}$ identifiées expérimentalement [11].

\begin{tabular}{cccccc}
\hline$k_{\theta_{1}}$ & $k_{\theta_{2}}$ & $k_{\theta_{3}}$ & $k_{\theta_{4}}$ & $k_{\theta_{5}}$ & $k_{\theta_{6}}$ \\
\hline $3,8 \times 10^{6}$ & $6,6 \times 10^{6}$ & $3,9 \times 10^{6}$ & $5,6 \times 10^{5}$ & $6,6 \times 10^{5}$ & $4,7 \times 10^{5}$ \\
\hline
\end{tabular}

Tableau 3. Caractéristiques mécaniques du T800 M21 (Hexcel).

\begin{tabular}{lc}
\hline Nombre de plis & 40 \\
Épaisseur des plis & $0,262 \mathrm{~mm}$ \\
Taux de fibres & $56,6 \%$ \\
Densité & $1580 \mathrm{~kg} \cdot \mathrm{m}^{-3}$ \\
Séquence de drapage & $\left(0^{\circ} / 0^{\circ} /-45^{\circ} / 0^{\circ} /-45^{\circ} / 90^{\circ} / 45^{\circ} / 0^{\circ} / 45^{\circ} / 0^{\circ} /\right.$ \\
& $\left.0^{\circ} / 0^{\circ} /-45^{\circ} / 0^{\circ} /-45^{\circ} / 90^{\circ} / 45^{\circ} / 0^{\circ} / 45^{\circ} / 0^{\circ}\right) 2 \mathrm{~s}$ \\
\hline
\end{tabular}

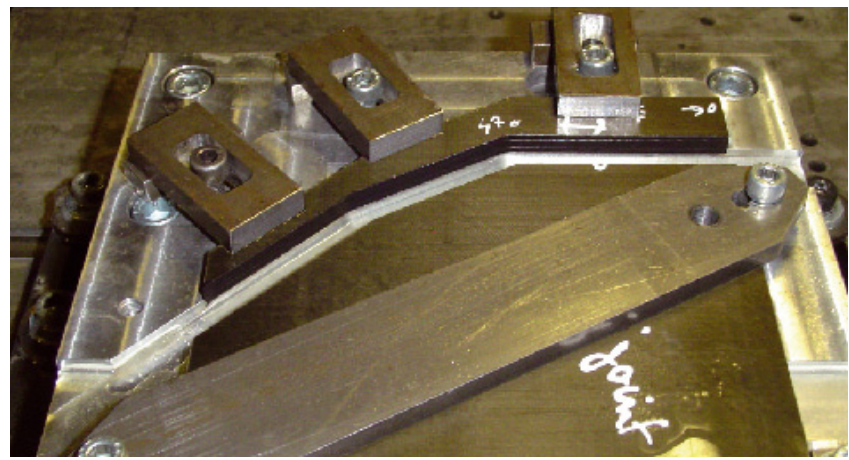

Fig. 3. Dispositif expérimental.

Tableau 4. Conditions de coupe sélectionnées suite aux essais préliminaires.

\begin{tabular}{cc}
\hline Vitesse de rotation & $27900 \mathrm{tr} \cdot \mathrm{min}^{-1}$ \\
Vitesse de coupe & $1400 \mathrm{~m} \cdot \mathrm{min}^{-1}$ \\
Vitesse d'avance & $6,4 \mathrm{~m} \cdot \mathrm{min}^{-1}$ \\
Avance par tour & $0,23 \mathrm{~mm} \cdot \mathrm{tour}^{-1}$ \\
Profondeur de passe & $10,5 \mathrm{~mm}$ \\
Largeur de passe & $16 \mathrm{~mm}$ \\
\hline
\end{tabular}

adaptés à notre application : d'un côté, ceux liés à l'outil (diamètre, granulométrie, nature des grains, taux de sertissage), de l'autre, ceux liés aux conditions de coupe (avance par tour, vitesse de coupe, direction des fibres par rapport à la vitesse d'avance). Suite aux différents essais, un outil de diamètre $16 \mathrm{~mm}$ et de granulométrie $852 \mu \mathrm{m}$ a été sélectionné. Des grains de diamants naturels ont été électro-déposés sur le corps de l'outil avec un taux de sertissage d'environ $50 \%$. Les conditions de coupe choisies sont présentées dans le tableau 4.

La trajectoire d'usinage a été choisie dans l'optique d'étudier l'influence de l'orientation des fibres sur les efforts de coupe. Trois rainures d'orientations différentes ont donc été réalisées. Les efforts moyens suivant les trois directions ont été mesurés (Fig. 4).

\subsubsection{Résultats expérimentaux}

Les efforts d'usinage associés aux paramètres opératoires précédemment définis ont été déterminés.

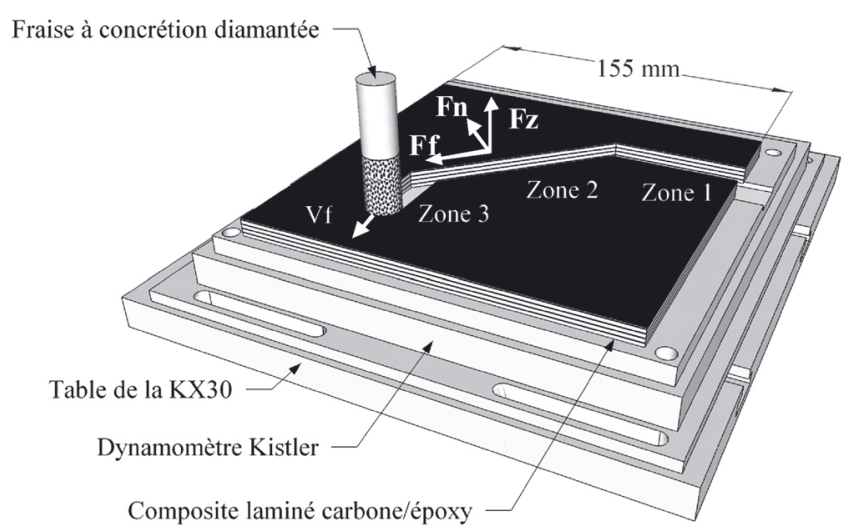

Fig. 4. Opérations d'usinage réalisées.

Tableau 5. Efforts moyens d'usinage.

\begin{tabular}{cccc}
\hline Orientation de la trajectoire & $F_{f}[\mathrm{~N}]$ & $F_{n}[\mathrm{~N}]$ & $F_{z}[\mathrm{~N}]$ \\
Zone $1: 0^{\circ}$ & 1056 & 373,1 & -20 \\
Zone $2: 45^{\circ}$ & 998 & 480 & $-40,9$ \\
Zone $3: 90^{\circ}$ & 1066 & 575 & $-19,9$ \\
\hline
\end{tabular}

Des essais de répétabilité ont validé la robustesse des résultats. Le tableau 5 présente les valeurs obtenues pour la trajectoire réalisée (Fig. 4). Les résultats montrent que les efforts d'usinage dépendent bien de l'orientation des fibres.

\section{Présentation du cas d'étude}

Afin de quantifier les défauts dimensionnels engendrés par les déformations du robot lors d'une opération d'usinage, le détourage d'une pièce de structure de grandes dimensions, de type aéronautique, a été étudié. Les résultats obtenus lors des essais préliminaires ont permis de quantifier les efforts nécessaires à la réalisation de cette pièce (Fig. 5).

Ayant connaissance d'une part du comportement du robot dans son espace de travail, et d'autre part des actions mécaniques mises en jeu durant l'usinage, il est maintenant possible de définir la trajectoire réelle du robot soumis aux différents chargements durant le cycle de détourage. 


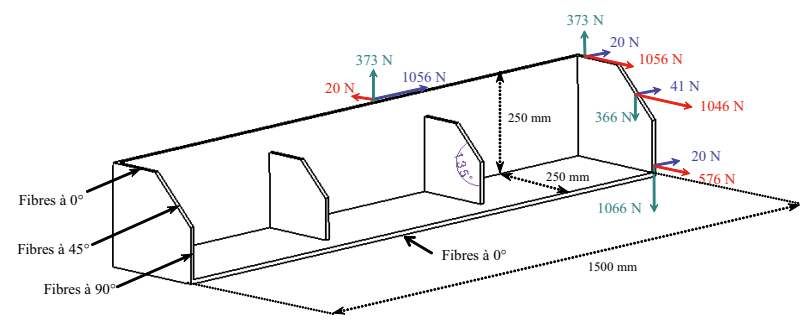

Fig. 5. Définition de la pièce et illustration des efforts d'usinage.

\section{Adaptation de la cellule robotisée en vue d'améliorer la qualité de la pièce détourée}

\subsection{Détermination de trois placements possibles de la pièce}

Plusieurs conditions doivent être respectées afin d'assurer un placement correct d'une pièce dans l'espace de travail du robot. Dans un premier temps, il est nécessaire que la trajectoire soit accessible, c'est-à-dire que le robot soit capable de faire coïncider un repère lié à son organe terminal avec un repère cible sans entrer en collision avec son environnement [25]. Dans un second temps, la trajectoire doit être T-parcourable de façon continue, c'està-dire parcourable sans changement brusque de configuration du robot. Dans notre cas d'application, il est envisageable que la trajectoire ne soit pas entièrement Tparcourable, mais il est nécessaire qu'elle le soit au moins par morceaux, c'est-à-dire que chacun des segments qui la composent soit T-parcourable.

Nous avons défini trois placements différents (Fig. 6) respectant les contraintes explicitées plus haut. Le premier placement est le plus « classique » : la pièce est posée à plat devant le robot. Dans le second cas, la pièce est mise « debout ». Enfin, la troisième position permet de limiter à la fois les mouvements du premier axe et la posture «bras tendu ».

\subsection{Détermination des déformations en bout du robot}

Les conditions de coupe retenues sont celles du tableau 4. Connaissant les raideurs articulaires du robot (Tab. 2), les efforts auxquels il est soumis ainsi que sa configuration, il est possible de prédire ses déplacements en translation et en rotation. Le tableau 6 présente les écarts calculés entre la trajectoire réelle et la trajectoire programmée, projetés dans le plan normal à la direction d'avance, ainsi que leurs valeurs moyennes et maximales pour l'ensemble des 2000 points de la trajectoire.

Ces valeurs permettent de classer les différents placements entre eux. Ainsi, le premier semble être le meilleur parmi les trois proposés, avec un écart moyen de 0,26 mm, donc inférieur à celui du placement 2 et relativement proche de celui du placement 3 . Il présente également l'écart maximal le plus faible. Afin d'expliquer cela, les déformations des articulations ont été analysées, celles des corps ayant une influence très faible sur la déformation en bout du robot [11]. Dans un premier temps les déformations en bout d'effecteur ont été recalculées en considérant les raideurs des trois premiers axes infinies (hypothèse du porteur rigide). Les résultats montrent que les déformations dues au seul poignet sont négligeables; elles ne peuvent pas expliquer les écarts calculés avec l'ensemble des flexibilités. Il convient donc dans un second temps d'analyser les déformations dues aux axes 1, 2 et 3 .

Le premier axe a une raideur comparable à celles des axes 2 et 3 (Tab. 2), alors que pour un effort de même intensité appliqué en bout du robot, cet axe doit résister à un couple plus important. Cela explique par exemple les différences de déformations, pour le placement 1, entre la trajectoire $\mathrm{P} 1$ à $\mathrm{P} 3$ et la trajectoire $\mathrm{P} 7$ à $\mathrm{P} 9$, pour lesquelles le bras était plus déplié (Tab. 6). De plus le placement 2 est celui qui sollicite le plus fortement l'axe 1 sur les trajectoires $\mathrm{P} 1$ à $\mathrm{P} 3$ et $\mathrm{P} 7$ à $\mathrm{P} 9$ par les efforts normaux à la direction d'avance $F_{n}$; les écarts moyens engendrés par ce placement sont donc les plus importants. Lorsque les efforts $F_{n}$ sont correctement orientés afin de limiter les sollicitations de l'axe 1 , il s'agit alors de limiter les sollicitations des axes 2 et 3 . Le placement 1 implique que le bras du robot soit plus déplié que pour le placement 3, excepté sur la trajectoire P10 à P12, ce qui explique que les déformations soient en moyenne plus importantes (l'axe 2 est soumis à un couple plus important), et que l'écart maximal soit obtenu pour le placement 3 .

Deux raisons expliquent donc les meilleurs résultats obtenus par le premier placement : un bras relativement replié, et des efforts normaux à la direction d'avance qui sollicitent l'axe 1 au minimum.

\section{Conclusion}

Les robots sont de plus en plus utilisés pour des opérations nécessitant à la fois raideur et précision. Sans étude du comportement du robot, il est impossible de pouvoir valider la faisabilité de la tâche. Effectivement, les déformations du robot peuvent être multipliées par dix pour une même opération réalisée à deux endroits différents de l'espace de travail. La géométrie de la pièce usinée dépend donc fortement de son placement. Le détourage des matériaux composites, utilisé comme exemple dans cet article, est une application mettant en jeu généralement des pièces de grandes dimensions. L'effecteur est également sollicité par des efforts importants. Dans ce cas, une bonne prise en compte de la déformation du manipulateur est primordiale. Une fois cette déformation évaluée, nous avons montré qu'il est possible, par des techniques ne nécessitant pas forcément la modification de la commande du robot, de la limiter par un meilleur placement de la tâche. Une optimisation multi-objectif peut ensuite être envisagée en couplant la diminution des sollicitations mécaniques dues au processus d'usinage et l'augmentation de la raideur effective du robot. 

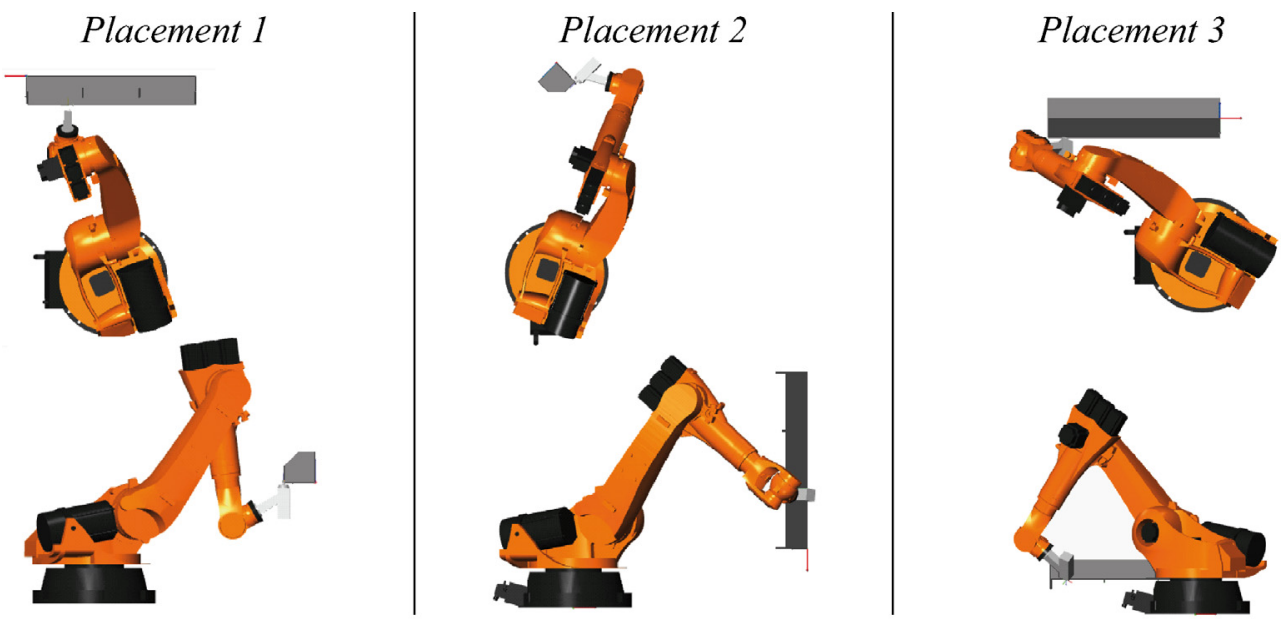

Fig. 6. Trois placements de la pièce dans l'espace de travail du robot.

Tableau 6. Écarts du bout d'outil le long de la trajectoire (porteur non rigide).

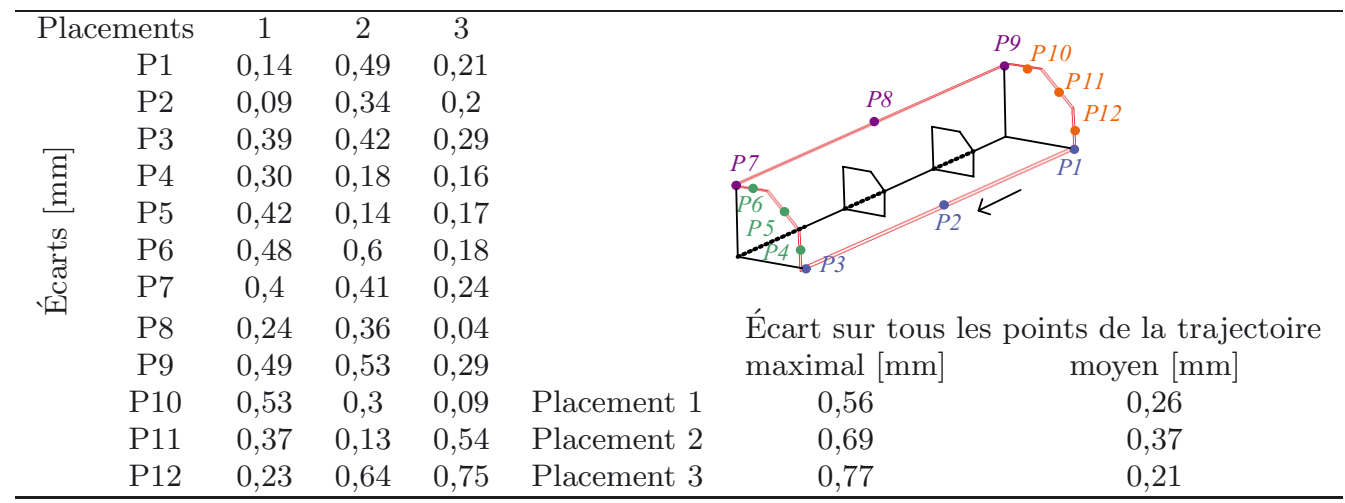

Remerciements. Nous remercions messieurs David Fontaine et Fabien Truchet, ingénieurs de recherche à l'IRCCyN, monsieur Philippe Moret, responsable du domaine Usinage à Hautes Performances chez Synervia (structure de transferts de technologies des Pays de la Loire), ainsi que monsieur Mehdi Cherif, maître de conférences au Laboratoire de Génie Mécanique et Matériaux de Bordeaux, pour son aide lors des essais expérimentaux.

\section{Références}

[1] B. Furet, B. Jolivet, D. Le Borgne, Milling and drilling of composite materials for the aeronautics: feature aeronautics, JEC Composite 18 (2005) 41-44

[2] M. Ramulu, D. Arola, Waterjet and abrasive waterjet cutting of unidirectional graphite/epoxy composite, Composites 24 (1993) 299-308

[3] D. Arola, M. Ramulu, A study of kerf characteristics in abrasive waterjet machining of graphite/epoxy composite, J. Eng. Mat. Technol. 118 (1996) 256-265

[4] M. Terrier, A. Dugas, J.-Y. Hascoet, Machines-outils à structure parallèle et usinage à grande vitesse, Mécanique \& Industries 6 (2005) 431-437
[5] S.-I. Matsuoka, K. Shimizu, N. Yamazaki, Y. Oki, Highspeed end milling of an articulated robot and its characteristics, J. Mater. Process. Technol. 95 (1999) 83-89

[6] H. Zhang, H. Hang, J. Wang, G. Zhang, Z. Gan, Z. Pan, H. Cui, Z. Zhu, Machining with flexible manipulator: toward improving robotic machining performance, Proc. IEEE/ASME International Conference on Advanced Intelligent Mechatronics, USA, 2005

[7] E. Abele, M. Weigold, S. Rothenbücher, Modeling and identification of an industrial robot for machining applications, CIRP Annals - Manufacturing Technology 56 (2007) 387-390

[8] A. Olabi, R. Bearee, M. Damak, O. Gibaru, Planification de l'avance sur trajectoire pour un robot 6 axes usinant, Colloque National AIP Primeca, La Plagne, France, 2009

[9] Z. Pan, H. Zhang, Z. Zhu, J. Wang, J. Chatter, Analysis of robotic machining process, J. Mater. Process. Technol. 173 (2006) 301-309

[10] S.-F. Chen, The $6 \times 6$ stiffness formulation and transformation of serial manipulators via the cct theory, IEEE International Conference on Robotics \& Automation, Taiwan, 2003

[11] C. Dumas, S. Caro, S. Garnier, B. Furet, A methodology for joint stiffness identification of serial robots, IEEE/RSJ International Conference on Intelligent Robots and Systems, Taiwan, 2010 
[12] K. Colligan, Edge trimming of graphite/epoxy with diamond abrasive cutters, ASME, Machining of Advanced Composite 45 (1992) 97-115

[13] J. Sheikh-Ahmad, Machining of polymer composites, Springer, 2009, pp. 164-165

[14] W. Konig, C. Wulf, P. GraßP., H. Willerscheid, Machining of fiber reinforced plastics, CIRP Annals - Manufacturing Technology 34 (1985) 537-548

[15] M. Ramulu, M. Faridnia, J.L. Garbini, J.E. Jorgenson, Machining of graphite/epoxy composite materials with polycrystalline diamond tools, J. Eng. Mater. Technol. 113 (1991) 430-436

[16] D.H. Wang, M. Ramulu, C.W. Wern, Orthogonal cutting characteristics of graphite/epoxy composite materials, Transactions of the NAMRI/SME 20 (1992) 159165

[17] Projet UGV aluminium-composites (2007), http://www .ugv-alu-composites .com

[18] K. Colligan, M. Ramulu, Edge trimming of graphite/epoxy with diamond abrasive cutters, J. Manuf. Sci. Eng. 121 (1999) 647-655
[19] A.J.P. Sabberwal, Chip section and cutting force during the milling operation, Ann. CIRP (1960) 197-203

[20] W.A. Kline, R.E. De Vor, The effect of runout on cutting geometry and forces in milling, Int. J. Mach. Tool Des. Res. 23 (1983) 123-140

[21] Y. Altintas, Manufacturing automation : metal cutting mechanics, machine tool vibration, and CNC Design, Cambridge University Press, New York

[22] H.Y. Feng, N. Su A Mechanistic cutting force model for 3d ball-end milling, ASME J. Manuf. Sci. Eng. 123 (2001) 23-29

[23] H.Y. Puw, H. Hocheng, Milling force prediction for fiber reinforced thermoplastics, Machining of Advanced Composites, Proc. ASME Winter Annual Meeting 45 (1993) 73-88

[24] J. Sheikh-Ahmad, R. Yadav, Force prediction in milling of carbon fiber reinforced polymers, Proc. IMECE, Orlando, 2005

[25] P. Chedmail, E. Dombre, P. Wenger, La CAO en robotique : outils et méthodologies, Hermès, 1998 